\title{
Duty of Care: Some Thoughts on The How? Where? and When?
}

Rehabilitation counsellors see duty of care for persons with illness, disability and disadvantage as a cornerstone of their professional identity. The Code of Ethics and the Core Competencies documents for the profession of rehabilitation counselling either directly relate to, or allude to, the need for adopting such an approach to each and every client with whom we come in contact. This granted, there appears to be some questions about the specifics of the duty of care we provide to clients to which we may offer differing responses.

- Where does duty of care begin and end?

- To what extent is our duty of care restricted to that period of time when the client is receiving rehabilitation services from us?

- Is the nature and time duration of duty of care determined by us individually?

- Is it a function of the relationship we have with the client?

- Is it indeed more about how the profession into which we have been drawn sees the boundaries for duty of care?

The accepted confines of duty of care for most rehabilitation counsellors are likely to be restricted to that period during which the person is a client of the rehabilitation counsellor and is actively receiving services (including follow-up services) from the counsellor. The nature and quality of the care provided to clients is often a function of the quality of the relationship that exists between the client and the counsellor. Whilst provision of differing levels of care is not to be applauded the fact that it happens simply recognises the relationship between some basic human traits and personality characteristics and others. Not everyone 'gels' with every other person ... that's why we don't all seek similar life partners ... its why we don't all gravitate to the same jobs. For the same reasons, not every client and every counsellor relate well to one another, and there is not always the luxury (especially in rural and remote regions) of transferring the case to another counsellor with whom the client may form a more productive relationship. As a result, the duration of duty of care may be similar; however, the quality of the care provided may be deficient.

Is there an alternate view of duty of care? To what extent is 'duty of care' a more encompassing concept which refers to a need for compassion, support and advocacy extended to our client base even before and after they are actual clients of ours. As individuals this more protracted and general approach to duty of care may not be feasible. However, as a group of professionals supported by a professional association we need to recognise that we have a power base, which can lobby on behalf of our current and future clients.

Why would we want to broaden the boundaries of our duty of care you may ask?? There are a number of reasons some altruistic and others economic. Surely we want to ensure that persons with disabilities have access to the best possible services and systems to allow them to live and work independently. We want to see that the range of services provided is expanded rather than contracted. We want to see that the 
whims of particular political persuasions do not override good policy, which deals with disability issues appropriately. That's the altruism aspect!

There is an economic advantage for us in ensuring that all that can be provided to persons with disability is indeed provided. As services are trimmed or streamlined, and as remuneration paid for specific services is capped or cut, our potential to ply our trade is significantly effected (and in a very negative way). That's the economic aspect!

The current orientation of political parties and policy makers is about reducing the financial burden of disability. Nothing wrong with that as a general concept. We would applaud attempts to allow more persons with disability access to the labour market. What is not applauded is the reduction in access to appropriate rehabilitation services or indeed access to appropriate monetary compensation for persons legitimately injured in accidents. What is also not applauded is the attempt to assess potential for work on a 'shoestring' budget. If people are to return to work after long periods of unemployment we have duty of care to adequately assess that potential.

Therefore, is it not the case that our duty of care extends beyond how, when, and what services we provide to the client when they come to us? Surely, our duty of care is about actively participating in debate about the nature of the mandated services that can be offered to the clients!! Our duty of care extends to caring about how injury is compensated, and what we will be funded to provide the person with the disability or the injured worker!

Trevor Hawkins, Guest Editor

University of Sydney 